\section{Characterization of Prophenoloxidase in Resisting Adverse Stresses in} Apis cerana cerana

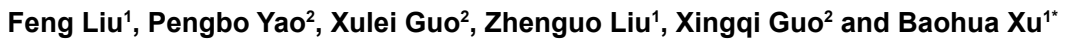 \\ ${ }^{1}$ College of Animal Science and Technology, Shandong Agricultural University, Taian, Shandong, 271018, P. R. China \\ ${ }^{2}$ State Key Laboratory of Crop Biology, College of Life Sciences, Shandong Agricultural University, Taian, Shandong, 271018, P. R. China
}

\begin{abstract}
Insect prophenoloxidases (PPOs) are a group of important innate immunity proteins and play an important role in melanin synthesis, defending the intruding microorganisms and parasites, healing wound and cuticle pigmentation. However, there is little study about immune-related defense mechanisms in Apis cerana cerana. Here we isolated a $P P O$ gene from $A$. cerana cerana and evaluated the connection of $A c c P P O$ to immunity. AccPPO has an open reading frame of 2079 bp encoding a 693 amino acid protein. The genomic structure analysis showed that AccPPO is similar to that of AmPPO. AccPPO is also close to AmPPO in the evolutionary period. AccPPO expression could be detected in all the developmental stages of $A$. cerana cerana and was the highest in 15-day postemergence adults. In addition, we also found that AccPPO was induced by the infection of Ascosphaera apis and various oxidative stresses including $4,16^{\circ} \mathrm{C}$; UV light; and pesticides (acaricide, cyhalothrin, paraquat) treatments. These results revealed that AccPPO may play a critical role in resisting Ascosphaera apis and preventing $A$. cerana cerana from oxidative stresses.
\end{abstract}

Keywords: Apis cerana cerana; quantitative real-time PCR; Ascosphaera apis; Adverse stresses

\section{Introduction}

Insect immunity is composed of cellular and humoral immunity. Cellular immunity is involved in circulating hemocytes that perform phagocytosis (of small bacteria) and encapsulation (around large parasites) [1]. While humoral immunity is induced through immunity proteins in hemolymph [2]. Insects depend on their innate immune system to protect against invasion by pathogens or parasites $[3,4]$. Insect prophenoloxidases (PPOs) are a group of important innate immunity proteins and are the zymogen of phenoloxidases (POs), which are generated by PPOs when insects are injuried and invaded by microbial or parasite [5]. Activated phenoloxidase (PO) can catalyse the formation of melanin during bacteria and parasite infections and melanin can encase and kill the invading pathogens [2,6]. POs, which catalyse the hydroxylation of monophenol to $o$-diphenol and its oxidation to $o$-quinone and $o$-quinone can generate in a nonenzymatic reaction $[7,8]$, function in melanin synthesis and are necessary for defense against intruding microorganisms and parasites, wound healing and cuticle pigmentation [9].

As the type 3 copper proteins, insect PPOshave a pair of copper atoms in the active site pocket and both of the copper atoms are coordinated by three histidine residues $[2,10]$. In Drosophila melanogaster, three PPO genes have been identified: PPO1 (CG5779), PPO2 (CG8193) and PPO3 (CG2952) [11]. PPO1 and PPO2 are primarily expressed in crystal cells while PPO3 is mainly expressed in lamellocytes when Drosophila melanogaster was infected by parasite [12-14]. Nine PPO genes have been identified in Anopheles gambiae $[15,16]$ while ten and three genes in Aedes aegypti and Culex quinquefasciatus, respectively [17]. In the honey bee Apis mellifera, the PPO cDNA was characterized [18].

Knockdown of PPO4 and other PPO transcripts in Anopheles dirus increased the prevalence of Plasmodium yoelii infection and the intensity of oocyst and abolished the melanization of oocysts [19]. In Aedes aegypti, PPO1, PPO3, PPO5, and PPO8 were involved in response to microbial infection [20]. In Culex pipiens pallens, $P P O A 3$ could not only be involved in immune defense, but plays an important role in resistance, suggesting that $P P O A 3$ might be an insecticideresistant associated gene [21]. Honey bee could be impacted when it was exposed to pesticides [22], which could also shorten the longevity of worker honey bee [23] and decrease the survival and weight of queen bees [24], and affect colony vitality [25]. There are also studies on PPO genes in other species, for example, $P P O$ was induced in all studied tissues after the chanllenge of $\mathrm{CpG}$ oligodeoxynucleotide (ODN), Aeromonas hydrophila and white spot syndrome virus (WSSV) [26].

Although the functions of PPO proteins have been characterized in other species, there is no research regarding the expression of $P P O$ gene when the organism is infected by parasite in the honey bee of A. mellifera and A. cerana cerana. The Chinese honeybee, A. cerana cerana is an importane indigenous species that plays an essential role in the balance of regional ecologies and agricultural economic development as the pollinator of flowering plants [27]. In addition, the survival environment is becoming more and more severe to A. cerana cerana due to the indiscriminate use of pesticides, infectious diseases and global warming [28]. It is important to characterize the resistant role of PPO protein in response to adverse environments in A. cerana cerana. Moreover, we also predicted that PPO might play an important role in resisting oxidative stresses. So we cloned and characterized the immune-related gene, $A c c P P O$, from A. cerana cerana and analyzed the genomic structure of $A c c P P O$. We also evaluated the expression profiles of $A c c P P O$ during different developmental stages using the quantitative real-time PCR (qRT-PCR) technology. At last, we investigated the transcripts of $A c c P P O$ in response to the infection of Ascosphaera apis and diverse oxidative stresses including $4,16^{\circ} \mathrm{C}$; UV light; and pesticides (acaricide, cyhalothrin, paraquat) treatments. Our results suggested that AccPPO might play an important role in defending the invasion of Ascosphaera apis. In addition, our data also first revealed that AccPPO may function in resisting oxidative stresses.

\section{Materials and Methods}

\section{Insects and treatments}

Colonies of A. cerana cerana at different developmental stages were collected from the experimental apiary of Shandong Agricultural

*Corresponding authors: Baohua $\mathrm{Xu}$, College of Animal Science and Technology, Shandong Agricultural University, Taian, Shandong, 271018, PR China, Tel: 86538-8241462; E-mail: bhxu@sdau.edu.cn

Received June 01, 2015; Accepted June 23, 2015; Published June 29, 2015

Citation: Liu F, Yao P, Guo X, Liu Z, Guo X et al. (2015) Characterization of Prophenoloxidase in Resisting Adverse Stresses in Apis cerana cerana. J Environ Anal Chem 2: 144. doi:10.4172/2380-2391.1000144

Copyright: ( 2015 Liu F, et al. This is an open-access article distributed under the terms of the Creative Commons Attribution License, which permits unrestricted use, distribution, and reproduction in any medium, provided the original author and source are credited. 
University (Taian, China). The fourth (L4), fifth (L5), and sixth (L6) day instar larvae, white-eyed $(\mathrm{Pw})$, pink-eyed $(\mathrm{Pp})$, brown-eyed $(\mathrm{Pb})$, dark-eyed (Pd) pupae and 1 day post-emergence adults (A1),15 day post-emergence (A15) and 30 day post-emergence adults (A30) were identified according to Yao et al. 2014. The worker honeybees of 15 day post-emergence adults were treated according to Yao et al. [29] with some modifications [30]. The larvae of $A$. cerana cerana were treated with Ascosphaera apis for 1,2 and 3 days, respectively, which was cultured in the larvae food to $1 \times 10^{6} \mathrm{cfu} / \mathrm{mL}$.

\section{Primers and PCR amplification conditions}

The primers and PCR amplification conditions are listed in Tables 1 and 2 , respectively.

\section{RNA extraction, cDNA synthesis and DNA preparation}

Total RNA (TansGen Biotech, Beijing, China) was extracted using Trizol reagent and the RNA samples were digested by the RNase-free Dnase I to remove potential genomic DNA. First-strand cDNA was generated by the EasyScript cDNA Synthetic SuperMix (TransGen Biotech, Beijing, China) according to the manufacture's protocol. The EasyPure Genomic DNA Extraction Kit (TransGen Biotech, Beijing, China) was used to isolate the Genomic DNA according to the manufacturer's instructions.

\section{Identification of AccPPO}

Primers PP1/PP2 were designed and synthesized (Biosune Biotechnological Company, Shanghai, China) based on the conserved region of $P P O$ genes from other species to gain the internal region of $A c c P P O$ cDNA. Using the sequence of obtained fragment, the 3' rapid amplification of cDNA ends (RACE) of AccPPO was performed using primers $3 \mathrm{P} 1 \mathrm{R} 1 / 3 \mathrm{P} 1 \mathrm{R} 2$. Then the partial $\mathrm{cDNA}$ sequence of $A c c P P O$ was obtained using primers QP1/QP2.

\section{Amplification of the genomic sequence of AccPPO}

To obtain the genomic DNA sequence of $A c c P P O$, primers N1/N2 were designed based on the obtained cDNA sequence of $A c c P P O$ and synthesized using the genomic DNA as the template. The PCR products were purified, cloned into pEASY-T3 vectors (TransGen Biotech, Beijing, China), and then transformed into competent Escherichia coli (E. coli) DH5a cells for sequence analysis.

\section{Bioinformatic analysis of AccPPO}

The procedures to conduct bioinformatic analysis of AccPPO were performed as previously described [31] with some modifications.

\section{Transcriptional profiling by qRT-PCR}

qRT-PCR was used to determine the expression level of AccPPO and primers TP1/TP2 were employed to amplify the a $137 \mathrm{bp}$ fragment of AccPPO. qRT-PCR was performed in $25 \mu \mathrm{L}$ reaction volume with the SYBR PrimeScript ${ }^{\mathrm{mi}}$ RT-PCR Kit (TaKaRa, Dalian, China) and a CFX96TM real-time PCR detection system (Bio-Rad). The amplification reaction protocol was performed as previous described. All samples were run in triplicate. The relative expression levels of $A c c P P O$ transcripts were determined using the $2^{-\triangle \Delta C T}$ method. Error bars denote the standard error of the mean (SEM) from three independent experiments. Significant differences were determined by Tukey HSD test using the SPSS software (Version 16.0, SPSS Inc). A p-value of 0.05 was used to determine statistical significance.

\section{Results}

\section{Characterization of AccPPO}

Based on the sequence information of $P P O$ genes in Apis mellifera, Apis dorsata, Apis florae, Bombus impatiens, Megachile rotundata, the partial cDNA sequence of $A c c P P O$ was cloned using a combination of reverse transcription PCR (RT-PCR) and rapid amplification of cDNA ends (RACE). The partial cDNA sequence of $A c c P P O$ was 2293 bp in length and contained a 27-bp 5' untranslated region (UTR), a 184-bp 3' UTR and an open reading frame of $2079 \mathrm{bp}$. The newly cloned AccPPO gene was predicted to encode a protein of 693 amino acid residues with

\begin{tabular}{|c|c|c|}
\hline Abbreviation & Primer sequence $\left(5^{\prime}-3^{\prime}\right)$ & Description \\
\hline PP1 & CATTCGACATCAGAACTATCG & cDNA sequence primer, forward \\
\hline PP2 & TTGCCATGTCAAGAGATATTC & cDNA sequence primer, reverse \\
\hline 3P1R1 & GTGGATTGAGAGATAGAAAGTATCC & 3' RACE forward primer, outer \\
\hline 3P2R2 & CGTGCAGGTGTTGAAACTTTAG & 3' RACE forward primer, inner \\
\hline B26 & GACTCTAGACGACATCGA $(T)_{18}$ & 3' RACE universal primer, outer \\
\hline B25 & GACTCTAGACGACATCGA & 3' RACE universal primer, inner \\
\hline QP1 & CATTCGACATCAGAACTATCG & Full-length cDNA primer, forward \\
\hline QP2 & TATTTTAACTATTCATTTATTTTTTTTTTTATTTTATTACAATAGA & Full-length cDNA primer, reverse \\
\hline$\beta-\mathrm{s}$ & TTATATGCCAACACTGTCCTTT & Standard control primer, forward \\
\hline$\beta-x$ & AGAATTGATCCACCAATCCA & Standard control primer, reverse \\
\hline N1 & CATTCGACATCAGAACTATCG & Genomic sequence primer, forward \\
\hline N2 & TATTTTAACTATTCATTTATTTTTTTTTTTATTTTATTACAATAGA & Genomic sequence primer, reverse \\
\hline TP1 & GGGTGATTCAGCAACAGCTATGAG & Real-time PCR primer, forward \\
\hline TP2 & CTATTTCGATACCCGGGAAGTC & Real-time PCR primer, reverse \\
\hline
\end{tabular}

Table 1: PCR primers in this study.

\begin{tabular}{|c|c|}
\hline Primes pair & Amplification conditions \\
\hline PP1/PP2 & $10 \mathrm{~min}$ at $94^{\circ} \mathrm{C}, 40 \mathrm{~s}$ at $94^{\circ} \mathrm{C}, 40 \mathrm{~s}$ at $50^{\circ} \mathrm{C}, 150 \mathrm{~s}$ at $72^{\circ} \mathrm{C}$ for 35 cycles, $10 \mathrm{~min}$ at $72^{\circ} \mathrm{C}$ \\
\hline 3P1B26 & $10 \mathrm{~min}$ at $94^{\circ} \mathrm{C}, 40 \mathrm{~s}$ at $94^{\circ} \mathrm{C}, 40 \mathrm{~s}$ at $50^{\circ} \mathrm{C}, 40 \mathrm{~s}$ at $72^{\circ} \mathrm{C}$ for 28 cycles, $10 \mathrm{~min}$ at $72^{\circ} \mathrm{C}$ \\
\hline 3P2B25 & $10 \mathrm{~min}$ at $94^{\circ} \mathrm{C}, 40 \mathrm{~s}$ at $94^{\circ} \mathrm{C}, 40 \mathrm{~s}$ at $53^{\circ} \mathrm{C}, 40 \mathrm{~s}$ at $72^{\circ} \mathrm{C}$ for $35 \mathrm{cycles}, 10 \mathrm{~min}$ at $72^{\circ} \mathrm{C}$ \\
\hline QP1/QP2 & $10 \mathrm{~min}$ at $94^{\circ} \mathrm{C}, 40 \mathrm{~s}$ at $94^{\circ} \mathrm{C}, 40 \mathrm{~s}$ at $51^{\circ} \mathrm{C}, 150 \mathrm{~s}$ at $72^{\circ} \mathrm{C}$ for $35 \mathrm{cycles}, 10 \mathrm{~min}$ at $72^{\circ} \mathrm{C}$ \\
\hline $\mathrm{N} 1 / \mathrm{N} 2$ & $10 \mathrm{~min}$ at $94^{\circ} \mathrm{C}, 40 \mathrm{~s}$ at $94^{\circ} \mathrm{C}, 40 \mathrm{~s}$ at $52^{\circ} \mathrm{C}, 240 \mathrm{~s}$ at $72^{\circ} \mathrm{C}$ for $35 \mathrm{cycles}, 10 \mathrm{~min}$ at $72^{\circ} \mathrm{C}$ \\
\hline
\end{tabular}

Table 2: $P C R$ amplification conditions. 


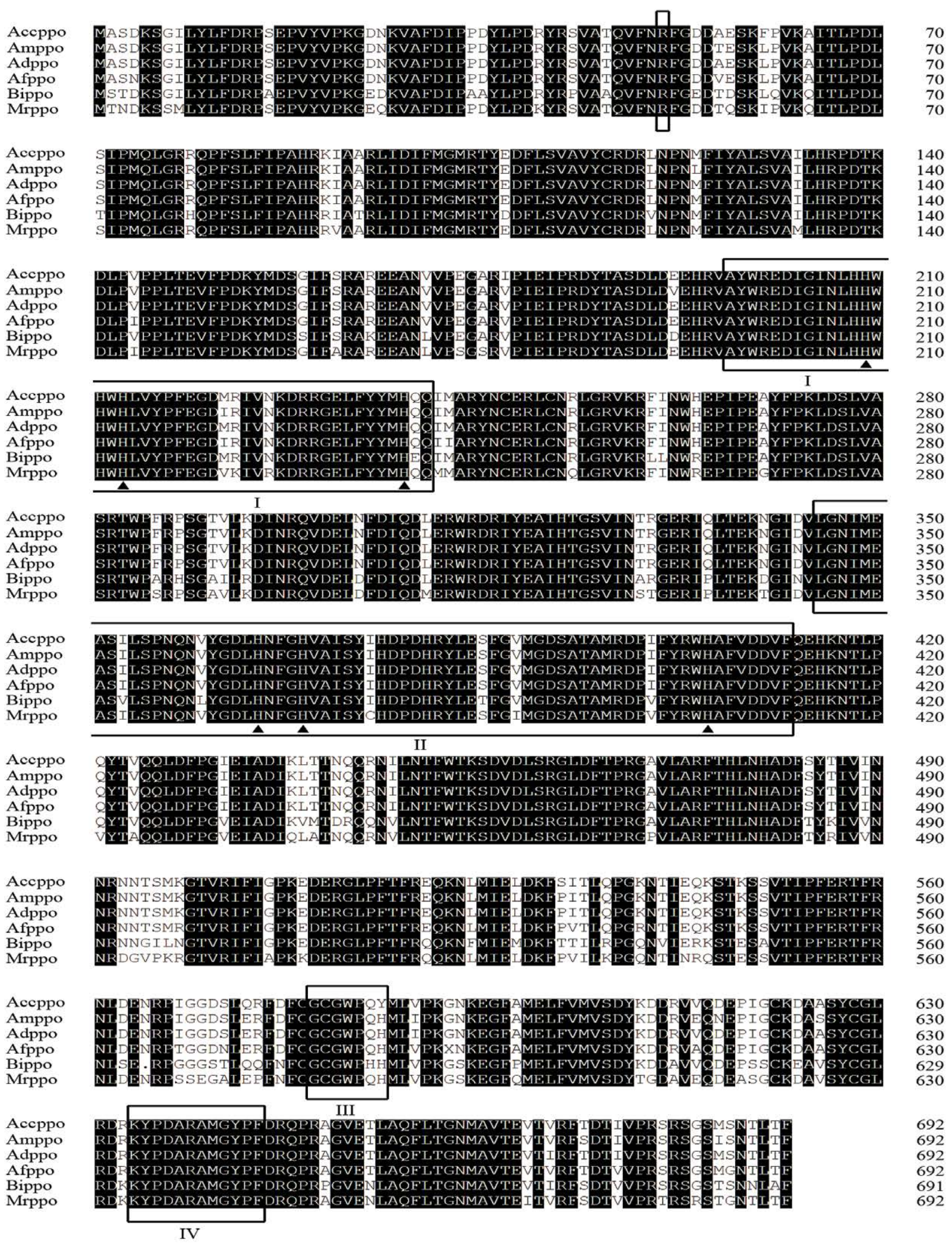

Figure 1: Molecular characterization of prophenoloxidases (PPOs) from various species. Comparison of the deduced amino acid sequences of AccPPO (prophenoloxidase, GenBank accession number: JX844653) from A. cerana cerana, AmPPO (prophenoloxidase, NP_001011627.1 from Apis mellifera, AdPPO (prophenoloxidase, XP 006616656.1) from Apis dorsata, AfPPO (prophenoloxidase, XP 003697839.1) from Apis florae, BiPPO (prophenoloxidase, XP_003484980.1) from Bombus impatiens and MrPPO (prophenoloxidase, XP_003707107.1) from Megachile rotundata. Identical amino acid residues are shaded in black. The predicted proteolytic cleavage site "R" is boxed. Conversed regions I, II, III and IV are also boxed. As shown in regions I and II, six histidine residues, which are considered to be ligands for the two copper atoms, are marked with $\boldsymbol{\Lambda}$. 
a putative molecular mass of $80.057 \mathrm{kDa}$ and an isoelectric point of 6.71. Multiple sequence alignments showed that the predicted amino acid sequence of AccPPO shares 97.84\%, 98.85\%, 97.26\%, 86.15\%, and $86.44 \%$ similarities with the PPO proteins from Apis mellifera, Apis dorsata, Apis florae, Bombus impatiens, and Megachile rotundata, respectively. As shown in Figure 1, the predicted proteolytic cleavage site " $R$ " is boxed and the predicted molecular weight of the active enzyme PO is $74.232 \mathrm{kDa}$. In addition, two conserved regions, the putative GCGWPQH/Y thiol ester site (region III) and the region IV are also boxed in (Figure 1). The regions I and II are two copper binding sites $(\mathrm{CuA}$ and $\mathrm{CuB})$. In addition, there are also six histidine residues, which are considered to be ligands for the two copper atoms.

\section{Phylogenetic analysis of AccPPO}

To further clarify the catalytic mechanism of AccPPO, the potential tertiary structure of AccPPO was constructed using SWISS-MODEL server, and the cysteines (His209, His213, His238, His365, His369 and His405) of catalytic cores were identified (Figure 2). To investigate the revolutionary relationship between AccPPO and its homologs, a phylogenetic tree was constructed and analyzed using MEGA (version 4.0) software. As shown in Figure 3, phylogenetic analysis revealed that AccPPO was closely related to the PPO protein from A. mellifera, which is in agreement with the relationship predicted from the multiple sequence alignment.

\section{Genomic structure analysis of AccPPO}

To further investigate the properties of $A c c P P O$, the genomic DNA sequence of AccPPO was obtained using the genomic DNA of $A$. cerana cerana for PCR amplification. The genomic DNA sequence of AccPPO (GenBank accession number: JX844654) was 3483 bp long, containing nine exons and eight introns, which is very similar to $A m P P O$ (Figure $4)$.

\section{Expression profiles of AccPPO in different developmental stages}

qRT-PCR was employed to analyse the mRNA accumulations of $A c c P P O$ during different developmental stages. As shown in Figure 5, the expression level of AccPPO could be detected in all the analysed developmental stages and it was expressed the highest in the 15-day postemergence adults.

\section{Expression patterns of AccPPO in response to oxidative stresses}

As shown above, AccPPO was expressed the highest in the 15day postemergence adults in different developmental stages and then 15-day postemergence adults were subjected to $4,16^{\circ} \mathrm{C}$; UV light; and pesticides (acaricide, cyhalothrin, paraquat) treatments. The expression level of $A c c P P O$ in larvae was induced by the infection of Ascosphaera apis (Figure 6A). As shown in (Figure 6B, 6C), when treated with cold stresses, the expression profiles of $A c c P P O$ was both induced under 4 and $16^{\circ} \mathrm{C}$ treatments and $A c c P P O$ was upregulated more obviously under $4^{\circ} \mathrm{C}$ treatment than that of $16^{\circ} \mathrm{C}$ treatment. At UV light treatment, the transcript of $A c c P P O$ was induced severely and reached 7.4-fold higher at $4 \mathrm{~h}$ in the treated groups compared with the control groups (Figure 6D). Under stress at acaricide, cyhalothrin and paraquat treatments, the expression levels of $A c c P P O$ were all induced by the above pesticides and reached their highest levels at $2.5 \mathrm{~h}, 1.0 \mathrm{~h}$ and $2.5 \mathrm{~h}$, respectively (Figures. 6E, 6F, 6G).

\section{Discussion}

Previous studies have shown that insect PPO plays an important role in immunity and the $\mathrm{PO}$ activity is essential to recover the damage caused by pathogen invasion and wounds [13]. There are also some researches about the function of PPO in resisting parasites in other species whereas there is little study on PPO in honey bee, particularly in Chinese honey bee (A. cerana cerana). To gain new insight into the functions of PPO in this important species, we identified and characterized a $P P O$ gene from A. cerana cerana in this study. In addition, we analyzed the expression profiles of $A c c P P O$ during different developmental stages and we also investigated the transcripts of $A c c P P O$ during various environmental stresses. Based on our results, we predicted that AccPPO might play a critical role in immunity and resisting oxidative stresses.

Sequence analysis showed that AccPPO has the same length ORF as AmPPO and also had the high amino acids identity to AmPPO, suggesting AccPPO might play the same important role in A. cerana cerana as that in A. mellifera. In addition, we could also find that the copper binding sites $\mathrm{CuA}$ and $\mathrm{CuB}$, the putative GCGWPQH/Y thiol ester site (region III) and the conserved region IV of AccPPO in A. cerana cerana were similar to those of AmPPO in A. mellifera, which further verified that AccPPO might function in immunity in $A$. cerana cerana. Genomic structure analysis revealed that $A c c P P O$ was very similar to $A m P P O$ and phylogenetic analysis of AccPPO and its homologs also showed that PPO protein in A. cerana cerana was close to that in A. mellifera, suggesting that AccPPO has a conserved period of evolution $[12,32]$.

It was not detected in pupae and expressed in other developmental stages in the lepidopteran Hyphantria cunea (H. cunea), which is not the same as that of $A c c P P O$ in A. cerana cerana. The PPOA3 gene of Culex pipiens pallens is transcribed at all developmental stages [31]. As shown in Figure 5, AccPPO was present in all of the developmental stages including the 4th-, 5th-, and 6th-day instar larvae; whiteeyed $(\mathrm{Pw})$, pink-eyed $(\mathrm{Pp})$, brown-eyed $(\mathrm{Pb})$, and dark-eyed $(\mathrm{Pd})$ pupae; 1-day postemergence adults; 15 -day postemergence and 30day postemergence adults, which is similar to that of $A m P P O$ in $A$. mellifera, revealing that AccPPO may also play a critical role in all the developmental stages. Moreover, the highest expression profile of $A c c P P O$ was found in 15-day postemergence adults among all the developmental stages, which is consistent with that of AmPPO in $A$. mellifer. In addition, Zhou et al. [11,31] demonstrated that the expression level of $P P O A 3$ gene from Culex pipiens pallens was higher in adult stage than other stages, suggesting they may play a more important role in immunity in adult stage.

Lourenco et al. [12] demonstrated that AmPPO functions in adult exoskeleton melanization in the previous study and PO-mediated melanin synthesis plays a major role in immune defense in insects. Melanin synthesis plays an important role in preventing hemolymph loss and providing cytotoxic compounds (quinone) to encapsulate and eliminate opportunist-invading microorganisms at the wound site $[32,33]$. The expression profile of $P P O$ was upregulated by after the stimulation of CpG ODN and WSSV in all studied tissues [11]. As shown in Figure 6, AccPPO was induced by the infection of Ascosphaera apis, revealing that AccPPO may function in preventing A. cerana cerana from the infection of Ascosphaera apis and play a major role in immunity, which is in agreement with previous studies. In addition, we could also surprisingly find that $A c c P P O$ was upregulated by some oxidative stresses including $4,16^{\circ} \mathrm{C}$; UV light; and pesticides (acaricide, cyhalothrin, paraquat) treatments, suggesting that AccPPO may also play an important role in resisting oxidative stresses. 


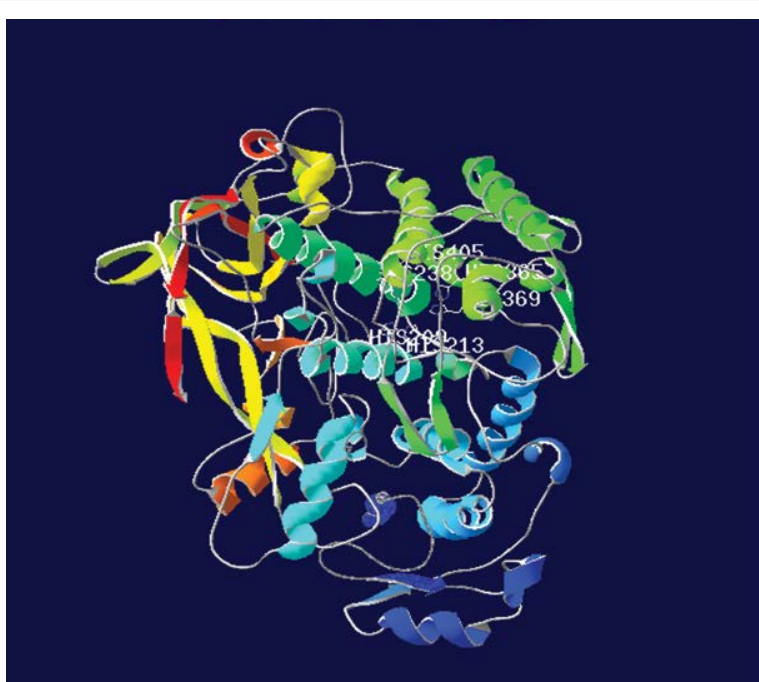

Figure 2: The tertiary structure of AccPPO. The structure was built using homology modeling in the SWISS-MODEL modeling environment. His209, His213, His238, His365, His369 and His405, which are found in the predicted catalytic active site, are shown

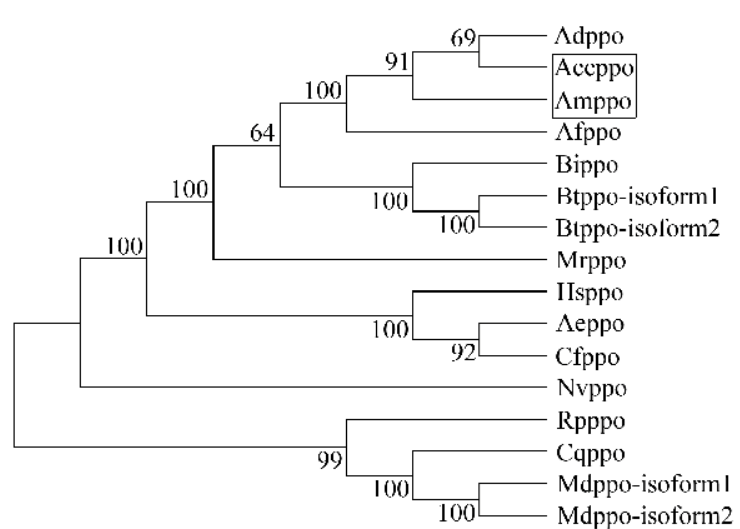

Figure 3: Phylogenetic analysis of $A c c P P O$ with other known PPO protein sequences. The protein sequences of various species are as follows: AccPPO (A. cerana cerana, GenBank accession number: JX844654) from A. cerana cerana AePPO (Acromyrmex echinatior, EGl66037.1), AdPPO (A. dorsata, XP 006616656.1), AfPPO (A. florae, XP 003697839.1), AmPPO (A. mellifera, NP_001011627.1), BiPPO (B. impatiens, XP_003484980.1), BtPPO isoform 1 (Bombus terrestris, XP_003400548.1), BtPPO isoform 2 (Bombus terrestris XP 003400549.1), CfPPO (Camponotus floridanus, EFN74080.1), CqPPO (Cūlex quinquefasciatus, XP 001848965.1), HsPPO (Harpegnathos saltator EFN88581.1), MrPPO (M. rotundata, XP_003707107.1), MdPPO isoform X1 (Musca domestica, XP 005174994.1), MdPPO isoform X2 (Musca domestica, XP 005174995.1), NvPPO (Nasonia vitripennis, XP 001606582.2), RpPPO (Riptortus pedestris, BAN20821.1).

In conclusion, we have identified an immune-related enzyme gene $(A c c P P O)$ from $A$. cerana cerana in this study. This enzyme possesses conserved functional domains of the PPO superfamilies and the genomic structure and phylogenetic tree of $A c c P P O$ are similar to that of $A m P P O$. The expression profile of AccPPO during different developmental stages was highest in 15-day postemergence adults. In addition, the expression level of AccPPO in response to the infection of Ascosphaera apis indicated that AccPPO may play a critical role in immune-related defense mechanisms. Very surprisingly, AccPPO was also induced by various environmental stresses. These findings provide solid evidence for the importance role of AccPPO in resisting the invasion of Ascosphaera apis and adverse environment to protect

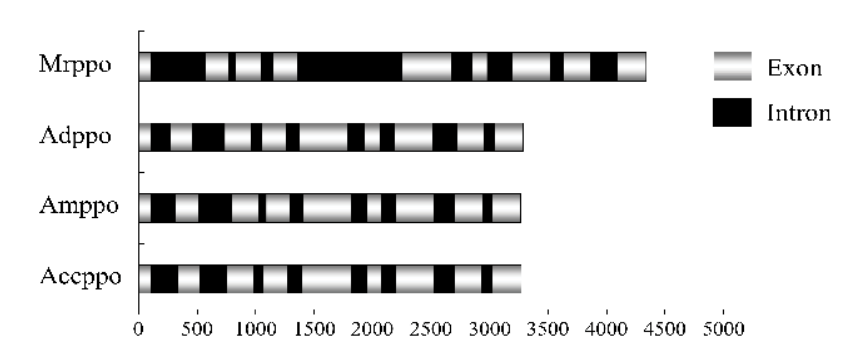

Figure 4: Genomic structure analysis of $A c c P P O$ from $A$. cerana cerana and its homologs, including AmPPO from A. mellifera (NP 001011627.1) MrPPO from M. rotundata (XP_003707107.1), and AdPPO from A. dorsata (XP_006616656.1).

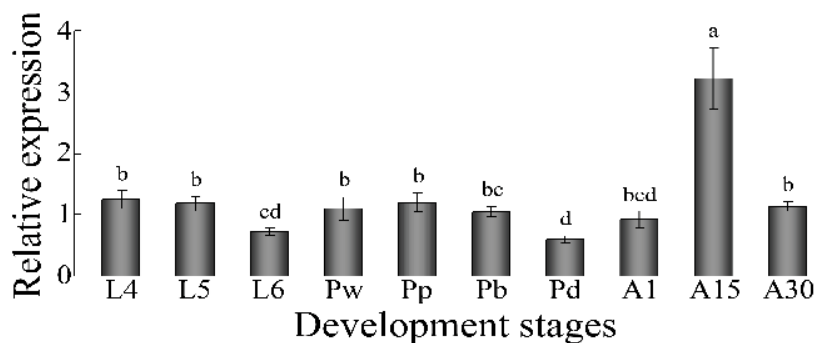

Figure 5: Expression patterns of $A c c P P O$ determined by real-time quantitative PCR. Expression profiles of AccPPO in all the developmental stages including larvae from the fourth to sixth instars ( $L 4-L 6$ ), pupae ( $P w$, white-eyed pupae; $\mathrm{Pp}$, pink-eyed pupae; $\mathrm{Pb}$, brown-eyed pupae; and $\mathrm{Pd}$, dark-eyed pupae), and adults (A1, 1 day postemergence; $A 15,15$ days postemergence; and $A 30$, 30 days postemergence). The $\beta$-actin gene was used as an internal control. Histograms reveal the relative expression profiles of AccTpx-3. Each value is given as the mean (Kohgo et al.) of three replicates. The letters on the bar represent a significant difference at $P<0.05$, as determined by Tukey HSD test using the SPSS software (Version 16.0, SPSS Inc).
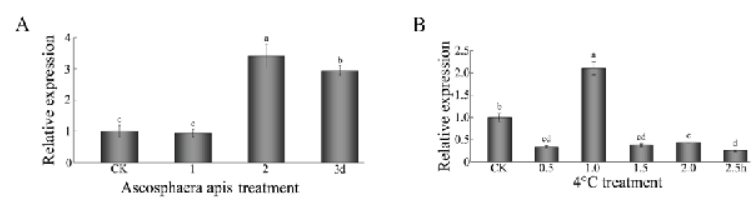

$\mathrm{C}$
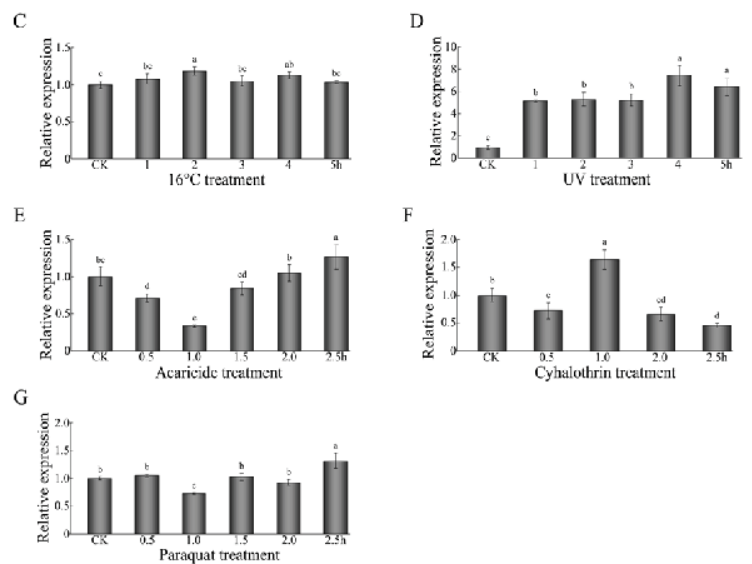

Figure 6: Effect of the infection of Ascosphaera apis and various oxidative stresses including $4,16^{\circ} \mathrm{C}$; UV light; and pesticides (acaricide, cyhalothrin, paraquat) treatments on AccPPO gene expression. The $\beta$-actin gene was used as an internal control. Histograms reveal the relative expression profiles of $A c c T p x-3$. Each value is given as the mean of three replicates. The letters on the bar represent a significant difference at $P<0.05$ as determined by Tukey HSD test using the SPSS software (Version 16.0, SPSS Inc). 
A. cerana cerana and enrich our understanding of PPO proteins in $A$. cerana cerana.

\section{Acknowledgments}

This work was financially supported by the earmarked fund for the China Agriculture Research System (No. CARS-45), the National Natural Science Foundation of China (No. 31172275) and Shandong Province Agricultural Fine Varieties Breeding Projects (2014-2016).

\section{References}

1. An C, Zhang M, Chu Y, Zhao Z (2013) Serine protease MP2 activates prophenoloxidase in the melanization immune response of Drosophila melanogaster. PLoS One 8: e79533.

2. Andersen S, Peter M, Roepstorff $P$ (1996) Cuticular sclerotization in insects Comp Biochem Physiol B Biochem Mol Biol 113: 689-705.

3. Ashida M, PT Brey (1998) Recent advances on the research of the insect prophenoloxidase cascade. In: Molecular Mechanisms of Immune Reponses in Insects. Chapman \& Hall, London, pp. 135-172.

4. Beliën T, Kellers J, Heylen K, Keulemans W, Billen J, et al. (2008) Effects of sublethal doses of crop protection agents on honey bee (Apis mellifera) global colony vitality and its potential link with aberrant foraging activity. Commun Agric Appl Biol Sci 74: 245-253.

5. Cerenius L, Lee BL, Söderhäll K (2008) The proPO-system: pros and cons for its role in invertebrate immunity. Trends Immunol 29: 263-271.

6. Christophides GK, Zdobnov E, Barillas-Mury C, Birney E, Blandin S, et al (2002) Immunity-related genes and gene families in Anopheles gambiae. Science 298: 159-165.

7. Irving P, Ubeda JM, Doucet D, Troxler L, Lagueux M, et al. (2005) New insights into Drosophila larval haemocyte functions through genome-wide analysis. Cell Microbiol 7: 335-350.

8. Iwanaga S, Lee BL (2005) Recent advances in the innate immunity of invertebrate animals. J Biochem Mol Biol 38: 128-150.

9. Kanost MR, Jiang H, Yu XQ (2004) Innate immune responses of a lepidopteran insect, Manduca sexta. Immunol Rev 198: 97-105.

10. Li X, Ma M, Liu F, Chen Y, Lu A, et al. (2012a) Properties of Drosophila melanogaster prophenoloxidases expressed in Escherichia coli. Dev Comp Immunol 36: 648-656.

11. Li Y, Deng W, Yang K, Wang W (2012) The expression of prophenoloxidase mRNA in red swamp crayfish, Procambarus clarkii, when it was challenged. Genomics 99: 355-360.

12. Lourenço AP, Zufelato MS, Bitondi MM, Simões ZL (2005) Molecula characterization of a cDNA encoding prophenoloxidase and its expression in Apis mellifera. Insect Biochem Mol Biol 35: 541-552.

13. Lu A, Peng Q, Ling E (2014) Formation of disulfide bonds in insect prophenoloxidase enhances immunity through improving enzyme activity and stability. Dev Comp Immunol 44: 351-358.

14. Mackenzie KE, Winston ML (1989) Effects of sublethal exposure to diazinon on longevity and temporal division of labor in the honey bee (Hymenoptera: Apidae). J Econ Entomol 82: 75-82.

15. Mason HS (1965) Oxidases. Annu Rev Biochem 34: 595-634.

16. Mason HS (1955) Comparative biochemistry of the phenolase complex. Adv Enzymol Relat Subj Biochem 16: 105-184.

17. Van dame R, Meled M, Colin ME, Belzunces LP (1995) Alteration of the homing-flight in the honey bee Apis mellifera L. Exposed to sublethal dose of deltamethrin. Environ Toxicol Chem 14: 855-860.

18. Pettis JS, Collins AM, Wilbanks R, Feldlaufer MF (2004) Effects of coumaphos on queen rearing in the honey bee, Apis mellifera. Apidologie 35: 605-610.

19. Strand MR (2008) The insect cellular immune response. Insect Sci 15: 1-14.

20. Sugumaran M (1998) Unified mechanism for sclerotization of insect cuticle. Advances in Insect Physiology 27: 229-334.

21. Tang H (2009) Regulation and function of the melanization reaction in Drosophila. Fly (Austin) 3: 105-111.

22. Wang Y, Hao H, Qiu ZW, Xu WY, Zhang J, et al. (2009) Involvement of prophenoloxidases in the suppression of Plasmodium yoelii development by
Anopheles dirus. Exp Parasitol 123: 6-10.

23. Wang Z, Lu A, Li X, Shao Q, Beerntsen BT, et al. (2011) A systematic study on hemocyte identification and plasma prophenoloxidase from Culex pipiens quinquefasciatus at different developmental stages. Exp Parasitol 127: 135141.

24. Waterhouse RM, Kriventseva EV, Meister S, Xi Z, Alvarez KS, et al. (2007) Evolutionary dynamics of immune-related genes and pathways in diseasevector mosquitoes. Science 316: 1738-1743.

25. Wertheim B, Kraaijeveld AR, Schuster E, Blanc E, Hopkins M, et al. (2005) Genome-wide gene expression in response to parasitoid attack in Drosophila. Genome Biol 6: R94.

26. Xu P, Shi M, Chen XX (2009) Antimicrobial peptide evolution in the Asiatic honey bee Apis cerana. PLoS One 4: e4239.

27. Yang G (2005) Harm of introducing the western honeybee Apis mellifera L. to the Chinese honeybee Apis cerana F. and its ecological impact. Acta Entomologica Sinica 48: 401-406.

28. Yao P, Lu W, Meng F, Wang X, Xu B, et al. (2013a) Molecular cloning expression and oxidative stress response of a mitochondrial thioredoxin peroxidase gene (AccTpx-3) from Apis cerana cerana. J Insect Physiol 59: 273-282.

29. Yao P, Hao L, Wang F, Chen X, Yan Y, et al. (2013) Molecular cloning expression and antioxidant characterisation of a typical thioredoxin gene (AccTrx2) in Apis cerana cerana. Gene 527: 33-41.

30. Yao P, Chen X, Yan Y, Liu F, Zhang Y, et al. (2014) Glutaredoxin 1, glutaredoxin 2 , thioredoxin 1 , and thioredoxin peroxidase 3 play important roles in antioxidant defense in Apis cerana cerana. Free Radic Biol Med 68: 335-346.

31. Zhou D, Hao S, Sun Y, Chen L, Xiong C, et al. (2012) Cloning and characterization of prophenoloxidase $\mathrm{A} 3$ (proPOA3) from Culex pipiens pallens. Comp Biochem Physiol B Biochem Mol Biol 162: 57-65.

32. Zufelato MS, Lourenço AP, Simões ZL, Jorge JA, Bitondi MM (2004) Phenoloxidase activity in Apis mellifera honey bee pupae, and ecdysteroiddependent expression of the prophenoloxidase mRNA. Insect Biochem Mol Biol 34: 1257-1268.

33. Zou Z, Shin SW, Alvarez KS, Bian G, Kokoza V, et al. (2008) Mosquito RUNX4 in the immune regulation of PPO gene expression and its effect on avian malaria parasite infection. Proc Natl Acad Sci U S A 105: 18454-18459. 